\title{
RELATO DE TÉCNICAS PARA PREPARO DE RUFIÕES EM CARNEIROS DESLANADOS
}

\author{
TEIXEIRA, Pedro Paulo Maia ${ }^{1}$ \\ OLIVEIRA, Maria Emília Franco ${ }^{1}$ \\ Moura, Ana Carolina ${ }^{2}$ \\ SANTOS, Victor José C. ${ }^{1}$ \\ LECHINOSKI, Lussandro C. ${ }^{2}$ \\ KZAM, Aline S. L. ${ }^{2}$ \\ RODRIGUES, Luiz Fernando de Souza ${ }^{2}$ \\ VICENTE, Wilter Ricardo Russiano ${ }^{1}$
}

RESUMO: O objetivo desse experimento foi relatar técnicas de rufiões, tempo de recuperação e influência na libido em carneiros Santa Inês. As técnicas de preparo foram: Aderência do pênis à parede abdominal (APPA $-\mathrm{n}=2)$, Estreitamento do óstio prepucial (EOP $-n=2$ ), fixação da curvatura caudal da flexura sigmóide (FCCFS - n=2), e desvio lateral do pênis (DLP $-\mathrm{n}=2$ ). Foram utilizados carneiros da Raça Santa Inês selecionados após um exame clínico geral do sistema reprodutor, além de teste de libido. As técnicas foram realizadas e após 20 dias da cirurgia, os animais foram submetidos ao teste da libido. A DLP apresentou-se menor tempo de recuperação cirúrgica, seguida pela FCCFS e APPA. Observou-se também que a EOP não apresentou confiabilidade por permitir a exposição do pênis. As técnicas não promoveram influência negativa no comportamento reprodutivo dos animais até o fim do experimento.

Palavras-chave: Rufião. Ovino.Técnicas. Libido.

SUMMARY: The aim of the present experiment was to report different teaser techniques, time to recover, and influence over the libido in male sheep. Four different techniques were compared: penis adherence to abdominal wall $(n=2)$, narrowing of the prepucial ostium $(n=2)$, fixation of the caudal sigmoid flexure $(n=2)$, and lateral shift of the penis $(\mathrm{n}=2)$. Santa Inês rams were selected after general clinical and genital apparatus examination. Twenty days after surgery, animals underwent libido's test. Surgical recovery was faster in LSP, followed by FCCSP and PAAW. NOP did not seem to be a reliable technique, once penis exposure occurred. All techniques did not play a role above breeding behavior of the animals until the completion of the study.

Keywords: Teaser. Sheep. Techniques. Libido.

RESUMEN: El objetivo de este experimento fue que informe técnicos rufianes, tiempo de recuperación y la influencia sobre la libido en caneros Santa Inés. Las técnicas de preparación fueron: La adherencia a la pared abdominal del pene (APAP - $\mathrm{n}=2$ ), estrechamiento del orificio prepucial $(\mathrm{EOP}-\mathrm{n}=2)$, la fijación de la curvatura caudal de la flexura sigmoidea (FCCFS - $\mathrm{n}=2$ ), y la desviación lateral pene (DLP $-\mathrm{n}=2$ ). Hemos utilizado los carneros de la raza Santa Inés seleccionado después de un examen clínico general del sistema reproductivo, y las pruebas de la libido. Las técnicas que se han realizado y después de 20 días de la cirugía, los animales fueron probados para la libido. El DLP fue menor el tiempo de recuperación quirúrgica, seguida de FCCFS y APPA. También se observó que el EOP no mostró fiabilidad al permitir la exposición del pene. Las técnicas no causó influencia negativa sobre el comportamiento reproductivo de los animales hasta el final del experimento.

Palabras clave: Rufianes. Las ovejas. Las técnicas. La libido.

\footnotetext{
${ }^{1}$ Faculdade de Ciências Agrárias e Veterinária (FCAV), Universidade Estadual Paulista (UNESP), Jaboticabal, Brasil.p_paulomt@yahoo.com.br.

${ }^{2}$ Centro de Pesquisa em Caprinos e Ovinos (CPCOP), Universidade Federal Rural da Amazônia (UFRA).
} 


\section{INTRODUÇÃO}

A detecção de cio ainda é um dos fatores limitantes para programas inseminação artificial e monta controlada na criação de ovinos de corte, e as biotécnicas da reprodução são fortes aliadas, tendo como objetivo de aumentar a eficiência reprodutiva e produtiva do rebanho, contribuir com o melhoramento genético e no controle de patologias do sistema reprodutivo (CARNEIRO, 2007; SIMPLICIO et al., 2007). Para minimizar esse empecilho exigiu-se o uso de machos incapacitados de fecundar, mas que efetuem saltos sobre as fêmeas, indicando o estro. Esses são denominados rufiões e podem ser obtidos através de diferentes métodos, sendo seu uso um método seguro e viável para detecção do cio e que, além disso, estimula o estro e a ovulação. (EURIDES et al., 1992).

O objetivo desse experimento foi relatar as seguintes técnicas de preparo de rufiões: aderência do pênis à parede abdominal (APPA), estreitamento do óstio prepucial (EOP), fixação da curvatura caudal da flexura sigmóide (FCCFS), e desvio lateral do pênis (DLP), mostrando sua aplicabilidade em machos ovinos da raça Santa Inês.

\section{MATERIAL E MÉTODOS}

Foram utilizados oito ovinos machos deslanados da Raça Santa Inês com idade variando de 12 a 18 meses e peso médio de $56,5 \mathrm{~kg}$.

Foram selecionados dois animais para cada técnica, após exame clínico geral, exame específico do sistema reprodutor, andrológico e de escala de pontuação de libido, adaptado de Price e Wallach (1991) e Chaves et al. (2002). Para estes últimos, os carneiros foram testados frente a um grupo de oito fêmeas, no qual duas estavam em estro induzido farmacologicamente, avaliando-os nos seguintes quesitos: reconhecimento das fêmeas em estro; olfação e perseguição insistente; cheiradas; cabeçadas; lambidas; reflexo de Flehmen; movimentos pélvicos; gotejados de sêmen; tentativa de monta; monta; serviço completo (cobertura) e tempo de recuperação (abaixo de dois minutos). Atribui-se um ponto por cada quesito, tendo os resultados dentro de uma escala de 1 a 12 pontos.

Para a realização das técnicas, os animais foram submetidos a um jejum hídrico e alimentar 24 horas antes do procedimento cirúrgico. Utilizou-se como sedativo o tranquilizante maleato de acepromazina a $1 \%^{3}$ na dose de $0,08 \mathrm{mg} / \mathrm{kg}$ de peso vivo, por via intramuscular. Para anestesia local realizou-se a infusão de cloridrato de lidocaína a $2 \%^{4}$, nas linhas de secção.

A técnica de EOP foi baseada na descrição de Vale et al. (2005) e Eurides e Silva (2002). Após tricotomia da região próxima ao óstio prepucial, anti-sepsia e preparo do campo operatório, expôs-se parte da bainha prepucial, seguido do pinçamento e exerese da porção exteriorizada, com bisturi 23 (Figura 1A e 1B). Suturou-se a mucosa da bainha à pele do prepúcio, em padrão simples separado, com categute 0 ( Figura 1C). Externamente, estreitou-se o óstio com duas suturas separadas de Wolf, com nylon 2-0. A retirada de

${ }^{3}$ ACEPRAN $1 \%$. Univet. Brasil.

${ }^{4}$ LIDOSTON®. Ariston. Brasil

Nucleus Animalium, v.4, n.2, nov.2012 
uma porção da bainha prepucial e o estreitamento externo tiveram como objetivo, diminuir o óstio prepucial causando fimose intencional. Ao final do procedimento, fixou-se uma sonda adaptada no óstio com o intuito de impedir futuras aderências, por um período de 5 dias (Figura 1D).

Figura 1

Técnica do EOP - A: Isolamento do campo operatório; B: pinçamento da bainha prepucial e secção; C: sutura com ponto simples separado; D: finalização da técnica com colocação da sonda no óstio prepucial.

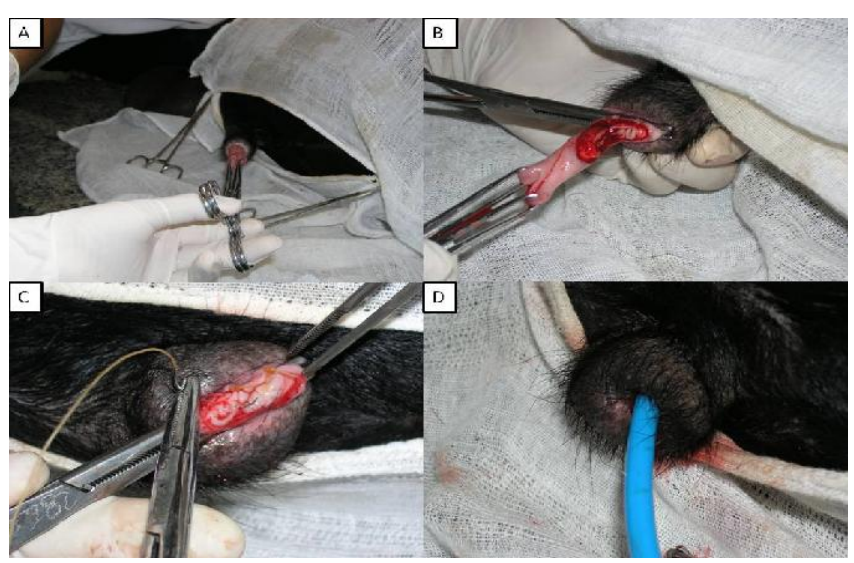

Fonte: Arquivo pessoal.

Conforme ao descrito por Castro et al (1994) e Eurides e Silva (2002), realizou-se a técnica de APPA após o preparo asséptico (como realizado na técnica anterior) da região ventral do abdome, cranialmente a base do escroto. Procedeu-se incisão de pele, de aproximadamente $10 \mathrm{~cm}$, com início na base do escroto, estendendo-a lateralmente ao prepúcio (Figura 1A). O corpo do pênis foi liberado da parede ventromedial do abdome, por meio de dissecação romba, exteriorizando-o através incisão. Após liberação do pênis dos tecidos adjacentes, a túnica albugínea da face dorsal do corpo do pênis e a musculatura ventromedial do abdome foram escarificadas com lâmina de bisturi 23. A porção dorsal do corpo do pênis e a parede ventromedial do abdome foram transfixadas pelos fios de sutura, com uma distância de 0,5 cm entre os pontos, aproximando estas estruturas (Figura 2B). A dermorrafia foi feita por meio de pontos Wolf separados, com fio de nylon 2-0 (Figura 2C e 2D). 
Figura 2: Técnica de APPA - A: Secção na linha lateral ao prepúcio para liberação do corpo peniano; B: fixação do corpo do pênis a parede abdominal; C e D: demorafia em Wolf separado.

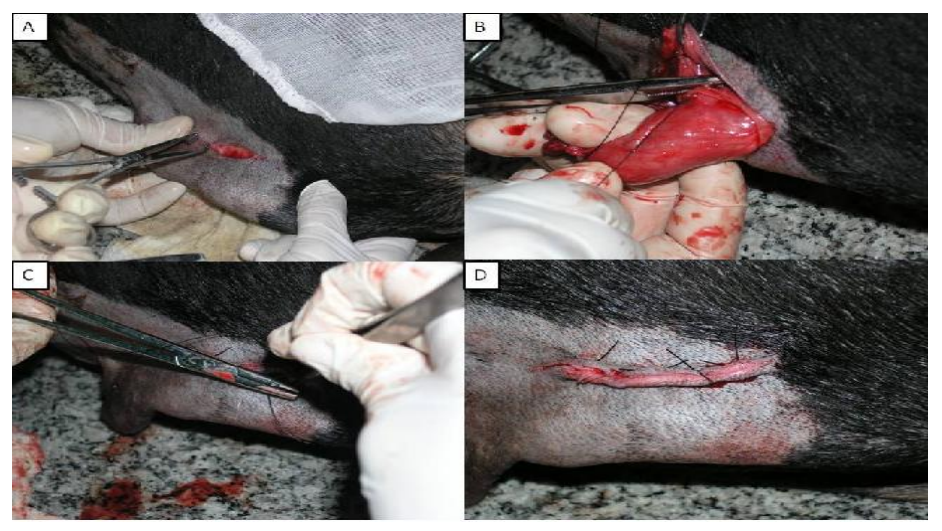

Fonte: Arquivo pessoal.

Semelhante descrita por Eurides e Pippi (1983) e Molinari et al. (1998), em bovinos, e Eurides et al, (1998), em ovinos, foi realizada a técnica de FCCFS. Após o preparo asséptico da região perineal, procedeu-se uma incisão na pele, acima da base do escroto com aproximadamente $5 \mathrm{~cm}$ de comprimento, com lâmina de bisturi 23 (Figura 3A). Por dissecação romba, foram separados o tecido subcutâneo e os músculos submembranosos até localizar a curvatura caudal da flexura sigmóide do pênis, próximo à base do escroto. Ainda com lâmina de bisturi 23, escarificou-se as faces da flexura (Figura 3B) e aproximou-as com dois pontos simples separados, com nylon 2-0, destacando o cuidado para não atingir a uretra peniana (Figura 3C). Realizou-se a redução do espaço morto com categute 1-0, e a dermorrafia com nylon 2-0, com pontos de Wolf separados (Figura 3D)

Figura 3: Técnica de FCCFS - A- Campo cirúrgico e perfusão de lidocaína na linha de secção; B- Escarificação das faces da flexura sigmóide; C- fixação com pontos simples separados; D- dermorrafia em pontos de Wolf separado.

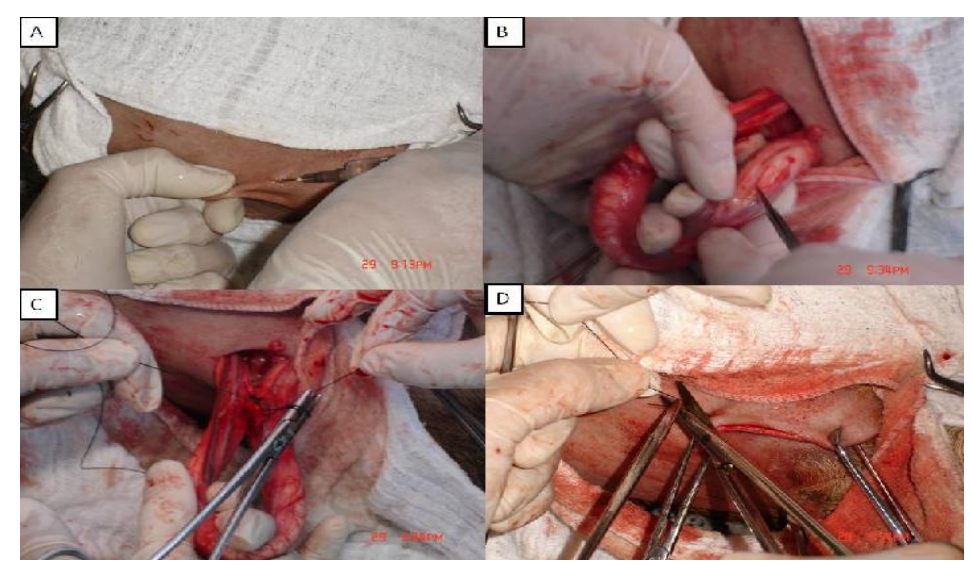

Fonte: Arquivo pessoal. 
A técnica de DLP foi realizada baseando-se em Nolasco et al. (2004), e Eurides e Silva (2002), em bovinos. Após o preparo asséptico em torno do prepúcio, na parede ventral do abdome, praticou-se uma incisão de pele, com lamina de bisturi 23, desde a extremidade cranial até o seu terço médio, envolvendo o tecido subcutâneo e o músculo cutâneo do tronco prepucial e músculo cutâneo prepucial cranial (Figura 4A e 4B). Outra incisão de pele e tecido subcutâneo foi realizada no antímero esquerdo, de tamanho aproximado ao anterior. O prepúcio foi transposto em um ângulo de $60^{\circ}$ e fixado com pinças de Allis na pele e no músculo cutâneo do tronco da parede abdominal. A pele da parede abdominal ventral, de onde foi retirado o prepúcio, foi aproximada e as bordas suturadas com fio de nylon 2-0, em sutura do tipo Wolff separado (Figura 4C). Finalmente, as bordas da pele do prepúcio foram suturadas na parede abdominal lateral, desde a angulação até o final da incisão também com os mesmos pontos e fio da sutura anterior (Figura 4D).

Figura 4: $\quad$ Técnicas de DLP - A- incisão da pele em volta do prepúcio; B- liberação do prepúcio e pênis; C- transplante para região lateral, fixação da pele com pontos em Wolf separado; D- curativo com unguento na ferida cirúrgica.

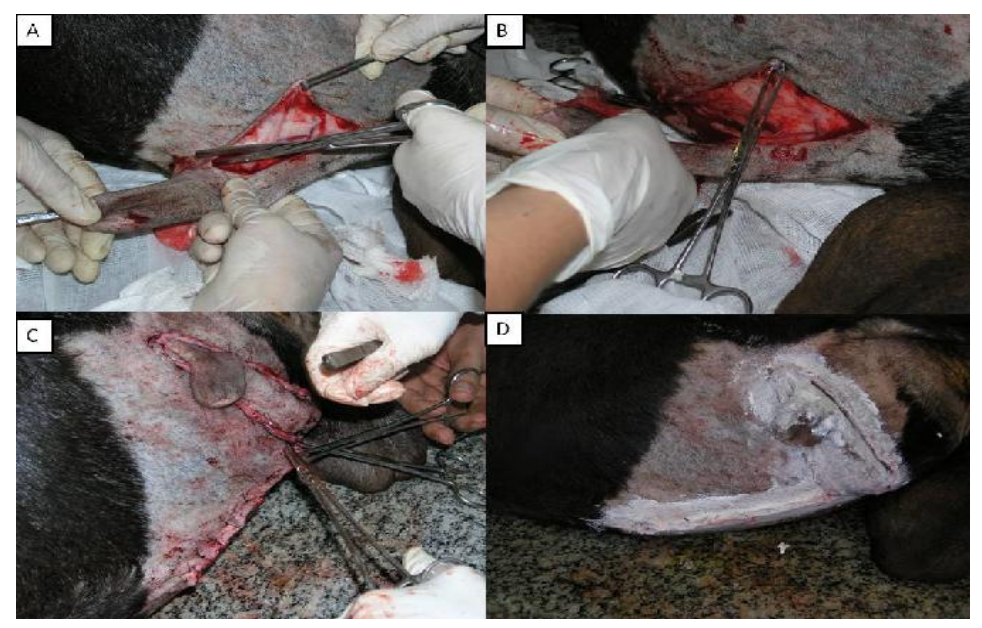

Fonte: Arquivo pessoal.

O pós-operatório constou de limpeza e curativos das feridas cirúrgicas e aplicação local de unguento e administração intramuscular de oxitetraciclina $20 \mathrm{~g}^{5}$, na dose de $0,1 \mathrm{ml} / \mathrm{Kg}$ de peso vivo. Após cinco dias os pontos foram retirados e aos 20 dias de pós-operatório os animais foram submetidos ao teste de libido novamente, com exceção da monta com serviço completo e tempo de recuperação, agora em uma escala de 1 a 10, sendo testados periodicamente por 2 anos. Para a análise dos resultados da escala de pontuação de libido empregou-se o teste one-way ANOVA (não paramétricos) empregado o pacote estatístico Grafped Prisma 4.

\footnotetext{
${ }^{5}$ Terramicina LA®, Pfizer, Brasil.
} 


\section{RESULTADOS E DISCUSSÃO}

Visibilizou-se que a técnica de EOP não apresentou confiabilidade por permitir, em um curto período ( 5 dias), a exposição do pênis, diferentemente do observado em bovinos e bubalinos por Vale et al. (2002). Eurides; Silva (2002) ressaltam que em técnicas de estreitamento do óstio prepucial, realizada em bovinos, a exposição de pênis é uma das complicações que podem ocorrer na técnica, podendo ser corrigida com uma neo-estenoses. Porém, pode causar dificuldade de micção e, nas tentativas de exposição do pênis, pode ocorrer deiscência da ferida no pós-operatório. Devido a não confiabilidade da técnica em ovinos, se faz necessário alterações na mesma, podendo realizar uma secção lateral antes da aproximação com os pontos de Wolf ou outra manobra cirúrgica que auxilie a não exposição do pênis.

As demais técnicas apresentaram eficiência na identificação de estro e monta, pois não permitiram a penetração e fecundação nas fêmeas, não necessitando de técnicas de esterilização, conforme cita Oehme (1988), Jochle et al. (1973), Royes e Bivin (1973) e Ostrowski e Rutter (1974), que recomendam a adição da vasectomia ou remoção da cauda do epidídimo às técnicas cirúrgicas que impedem a cópula, para assegurar a ausência de prenhez caso haja cópula acidental.

Observou-se que carneiros na faixa etária de 12 e 24 meses apresentaram-se aptos na detecção de estro após processos cirúrgicos de rufiões, semelhante ao descrito por Tharp (1955), Royes; Bivin (1973) e Eurides (1981), utilizando bovinos após a puberdade, oposto de Belling (1961); Jochle et al. (1973) que recomendam a utilização de animais antes da puberdade.

Pôde-se verificar que em relação ao gasto farmacológico, não houve considerável diferença entre as técnicas, visto que foram necessários os mesmos medicamentos tanto no pré como no pós-operatório, com diferença irrisória no anestésico local e fio de sutura. Considerando o tempo gasto para a realização das técnicas, em uma escala crescente teve a seguinte ordem: FCCFS, APPA, EOP e DLP.

Quanto ao tempo de recuperação cirúrgica, observou-se que a DLP apresentou melhor resultado, visto que com cinco dias os animais apresentaram-se completamente recuperados do processo cirúrgico e prontos para utilização, seguidos pela FCCFS e APPA (ambos em 10 dias). Vale ressaltar o aparecimento de edema na região prepucial nos animais submetidos à técnica de APPA.

Pelos testes da libido, ao longo de dois anos, pode-se comprovar que as técnicas de DLP e FCCFS não promoveram influência negativa no comportamento reprodutivo, no entanto ( $>>0,05)$, para técnica de APPA observou-se uma discreta diminuição somente no $24^{\circ}$ mês de avaliação $(\mathrm{p}<0,001)$ (Tabela 1). 
Tabela 1: Resultado da escala de pontuação durante os 24 meses de avaliação dos rufiões.

\begin{tabular}{cccc}
\hline Tempo em meses & APPA & FCCFS & DLP \\
\hline 0 & 10,0 & 10,0 & 10,0 \\
2 & 10,0 & 10,0 & 10,0 \\
4 & 10,0 & 10,0 & 10,0 \\
6 & 10,0 & 10,0 & 10,0 \\
8 & 10,0 & 10,0 & 10,0 \\
10 & 10,0 & 10,0 & 10,0 \\
12 & 9,5 & 10,0 & 10,0 \\
18 & 9,0 & 10,0 & 10,0 \\
24 & $8,5^{*}$ & 10,0 & 10,0 \\
\hline$* \mathrm{P}<0,001$, mostrado alteracão na libido com 24 meses.
\end{tabular}

* $\mathrm{P}<0,001$, mostrado alteração na libido com 24 meses.

Esses resultados conflitam com os achados de Shipilov (1964), Eurides e Pippi (1983), Straub e Kendrick (1965) que relataram que técnicas de rufiões que impedem a cópula podem influenciar psicologicamente a libido desses machos, diminuindo com o passar do tempo, devido à impossibilidade de copular. No entanto, concordando com Merkt e Sampaio (1973) e Eurides et al. (1998), que relataram que o interesse sexual dos rufiões bovinos submetidos ao desvio lateral do pênis, manteve-se durante todo o período de observação, e que em rufiões ovinos preparados pela técnica de retroflexão peniana, não apresentaram alteração na libido por 12 meses em que foram avaliados, resultado também verificado por Eurides e Pippi (1983) com fixação da curvatura caudal da flexura sigmóide do pênis.

\section{REFERENCIAS}

BELLING, T.H. Preparation of a "Teaser" bull for use in a beef cattle artificial insemination program. Journal of Americam Veteriray Medycal Association, v.138, n.12, p. 670-672, 1961..

CARNEIRO, G.F. Biotecnologia da reprodução na espécie caprina: perspectivas atuais. Revista Brasileira de Reprodução Animal, Belo Horizonte, v.31, n.2, p.268-273, 2007.

CASTRO, M.A.S.; MARÇAL, A.V.; MIDON FILHO, I. Preparo de rufiões bovinos pela fixação do corpo do pênis na Parede ventro-medial do abdome. Revista da FZVA. Uruguaiana, v.1, n.1, p.52-59, 1994.

CHAVES, S.M. Avaliação do Comportamento Sexual de Rufiões Bovinos Preparados através do desvio lateral modificado e da aderência do pênis à parede abdominal. Ciência Animal Brasileira. v.3, n.2, p.65$72,2002$.

EURIDES, D.; SILVA, L.A. Métodos de preparo de rufiões bovinos e equinos. Goiânia: Talento, 2002.

EURIDES, D. Preparação de rufiões ovinos por fixação da curvatura caudal da flexura sigmóide do pênis. Revista Brasileira de Ciência Veterinária, v.5, n.2, p.59-62, 1998.

EURIDES, D.; CONTESINI, E.A.; VIANA, S.M. Preparação de rufiões bovinos por remoção do ligamento apical do pênis. Ciência Rural, Santa Maria, v.22, n.2, p.185-189, 1992.

EURIDES, D.; PIPPI, N.L. Preparação de rufiões bovinos por fixação da flexura sigmóide do pênis. Revista do Centro de Ciências Rurais, Santa Maria, v.13, n.1, p.83-93, 1983. 
JOCHLE, W. Preparation of teaser rams boara by penis and prepuce desviation. Veterinay Medicine Small Animal Clincal, v.68, n.4, p.395-400, 1973.

MERKT, J.G.; SAMPAIO, F.A. Suchbullen durch penisablenkung nach operativer verlagerung des praeputiums. Dtsch tierärztl Wschr, v.80, n.22, p.515-519, 1973..

MOLINARI, C.E.; HRDALO, J.C.; GIL, O.H. Fijacion de la flexura distal de la S sigmoidea para la preparacion de toros marcadores de celo (tecnica de Monroy). Nuestra experiencia VeterinariaArgentina, v.15, n.142, p.109-113, 1998.

NOLASCO, R.M. Preparo de rufião bovino por desvio lateral do prepúcio em $90^{\circ} \mathrm{com}$ a linha mediana ventral do abdome. Ciência Animal Brasileira. v.5, n.2, p. 93-97, 2004.

OEHME, F.W. Textbook of large animal surgery. 2.ed. Baltimore: Williams \& Wilkins, 1988. 714p.

OSTROWSKI, J.E.B.; RUTTER, B. Surgical lateral displacement of the penis of bull's to be used teaser. Gaceta Vet. v.36, n.293, p. 652-660, 1974.

PRICE, E.O.; WALLACH, S.J.R. Inability to predict the adult sexual performance of bulls by prepuberal sexual behaviors. Journal of Animal Science, v.69, p.1041-1046, 1991.

ROYES, B.A.P.; BIVIN, S. Surgical dispacement of the penis in the bull. Journal American Veterinary Medical Association, v.163, n.1, p. 56- 57, 1973.

SHIPILOV, V.C. Surgical method for the permanent lateral fixation of the bull's prepuce and penis. Veterinary, v.41, n.8, p. 60-64, 1964.

SIMPLÍCIO, A.A.; FREITAS, J.V.F.; FONSECA, J.F. Biotécnicas da reprodução como técnicas de manejo reprodutivo em ovinos. Revista Brasileira de Reprodução Animal, v.31, n.2, p. 234-246, 2007.

STRAUB, O.C.; KENDRICK, J.W. Preparation of teaser bulls by penectomy. Journal American Veterinary Medical Association, v.147, n.4, p.373-376, 1965.

THARP, V.L. Vasectomy in the bull. Journal American Veterinary Medical Association, v.126, n.935, p. 96-99, 1955.

VALE W.; RIBEIRO, H.; ROLIN, S. Técnica de Estreitamento do óstio prepucial em bovinos e bubalinos. Descrição Pessoal Universidade Federal Rural da Amazônia, Brasil. 2005. 